\title{
Spectroscopy of white dwarf candidates from the ESO Imaging Survey (EIS-WIDE)^
}

\author{
D. Koester and B. Wolff
}

\author{
Institut für Theoretische Physik und Astrophysik, Universität Kiel, 24098 Kiel, Germany \\ e-mail: wolff@astrophysik.uni-kiel.de
}

Received 30 March 2001 / Accepted 6 September 2001

\begin{abstract}
In the ESO Imaging Survey of a 1.27 square degree field in the direction of the south galactic pole ("patch B") 32 point sources were identified as white dwarf candidates in the $B-V$ vs. $V-I$ two-color diagram by the EIS team and published as a candidate catalog. One of these was a previously known quasar. We have obtained low resolution spectra of 30 of the remaining sources. Of these only 6 are DA white dwarfs, one is a DQ white dwarf, one a DC. 11 candidates are quasars with redshifts between 1.4 and 3.3. The remaining sources are stars with narrow Balmer lines, in most cases probably field horizontal branch stars.
\end{abstract}

Key words. stars: white dwarfs - quasars: general

\section{Introduction}

The ESO Imaging Survey (EIS) was a small public survey conducted by the European Southern Observatory (ESO) before the advent of the first VLT telescopes. Its aim was to provide targets for further spectroscopic observations with the new large instruments. It consisted of two parts (Renzini \& Da Costa 1997): EIS-deep for defining samples of high-redshift galaxies and EIS-wide for the search of rare objects like distant clusters of galaxies. The latter was a wide-angle survey of four patches of the sky chosen to cover a range of galactic latitudes (Nonino et al. 1999). One of these patches, "patch B", points to the south galactic pole and has been observed in the three filters $B, V$, and $I$. As one of the byproducts Zaggia et al. (1999) have identified a small cata$\log$ of 32 white dwarf candidates in this 1.27 square degree field. The list is publicly available on the EIS webpage (http://http.hq.eso.org/science/eis/).

Although this field is relatively small and the number of candidates limited, the catalog could nevertheless be useful for the study of statistical properties of the white dwarf population, provided that a significant fraction of this catalog could be confirmed as white dwarfs. Because of its well defined selection criteria and homogeneous data, it is complementary to the typical very

Send offprint requests to: D. Koester, e-mail: koester@astrophysik.uni-kiel.de

* Based on observations collected at the European Southern Observatory, Paranal, Chile (ESO Programme 65.H-0008).

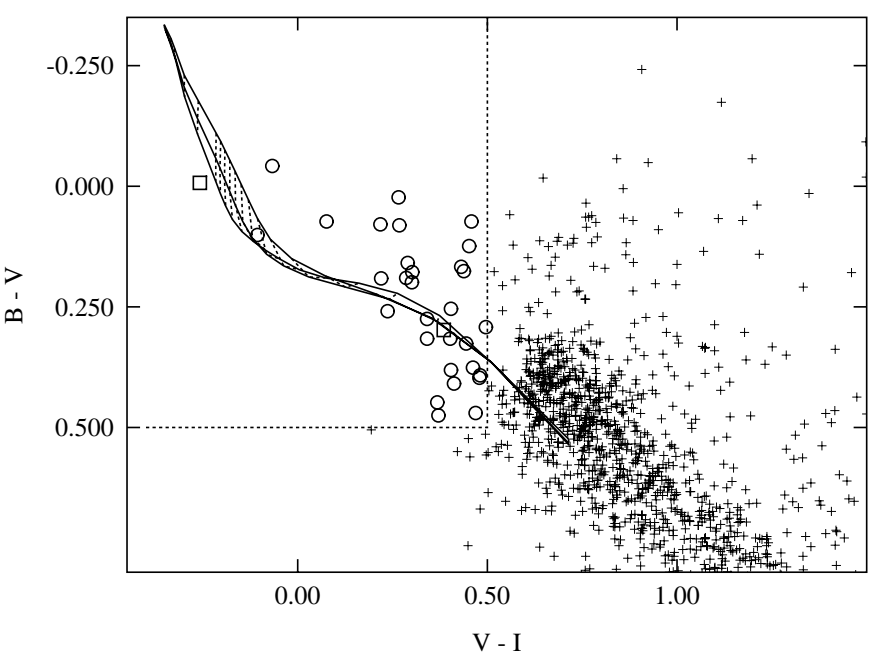

Fig. 1. BVI two-color diagram of point sources (crosses) in the EIS-WIDE patch B. Shown here are only the bluest objects in the upper left corner of the diagram. Circles are white dwarf candidates as selected by the color criteria shown by the dotted straight lines. The continuous and dotted curves are theoretical colors for DA white dwarfs. The two small squares are the two known (WD_28) resp. suspected (WD_25) quasars among the white dwarf catalog objects.

large area surveys of blue objects or high proper motion stars with limiting magnitudes in the range of $V=15-$ 18, which have in the past been used for such studies, e.g. the Palomar-Green Survey (Green et al. 1986), or 

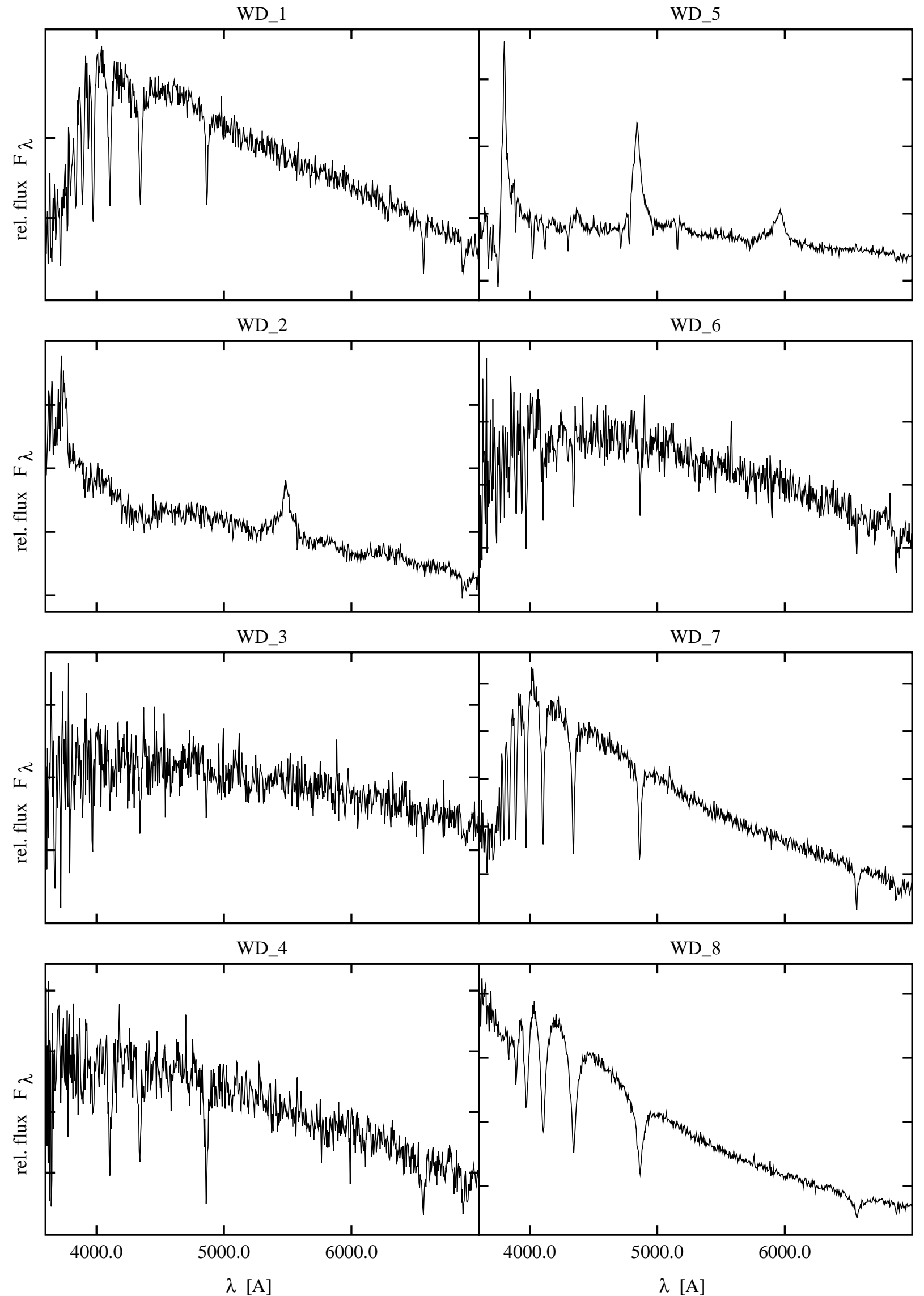

Fig. 2. VLT/FORS1 spectra of white dwarf candidates. The vertical scale is the relative intensity on an arbitrary linear scale. 

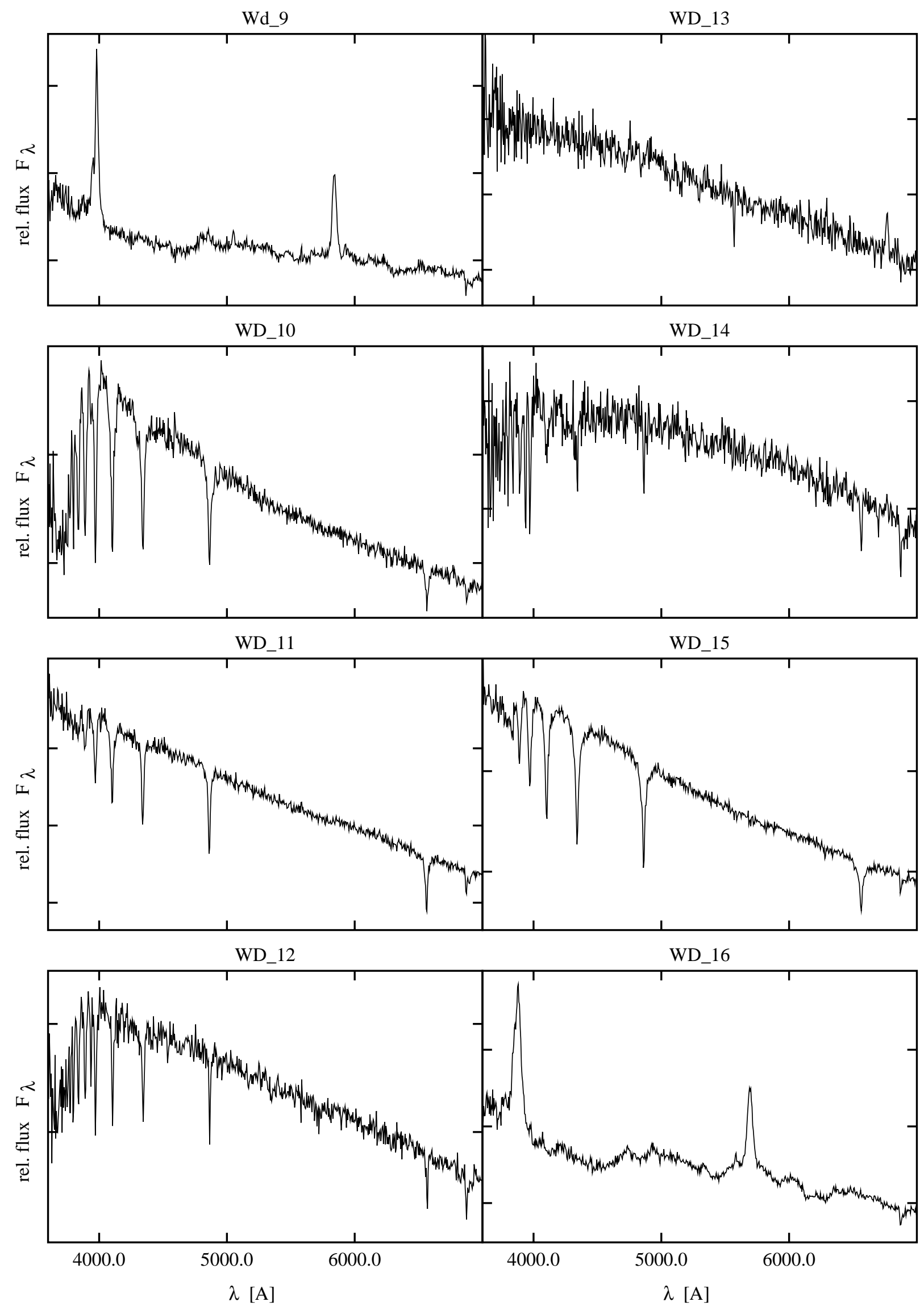

Fig. 3. VLT/FORS1 spectra of white dwarf candidates. 

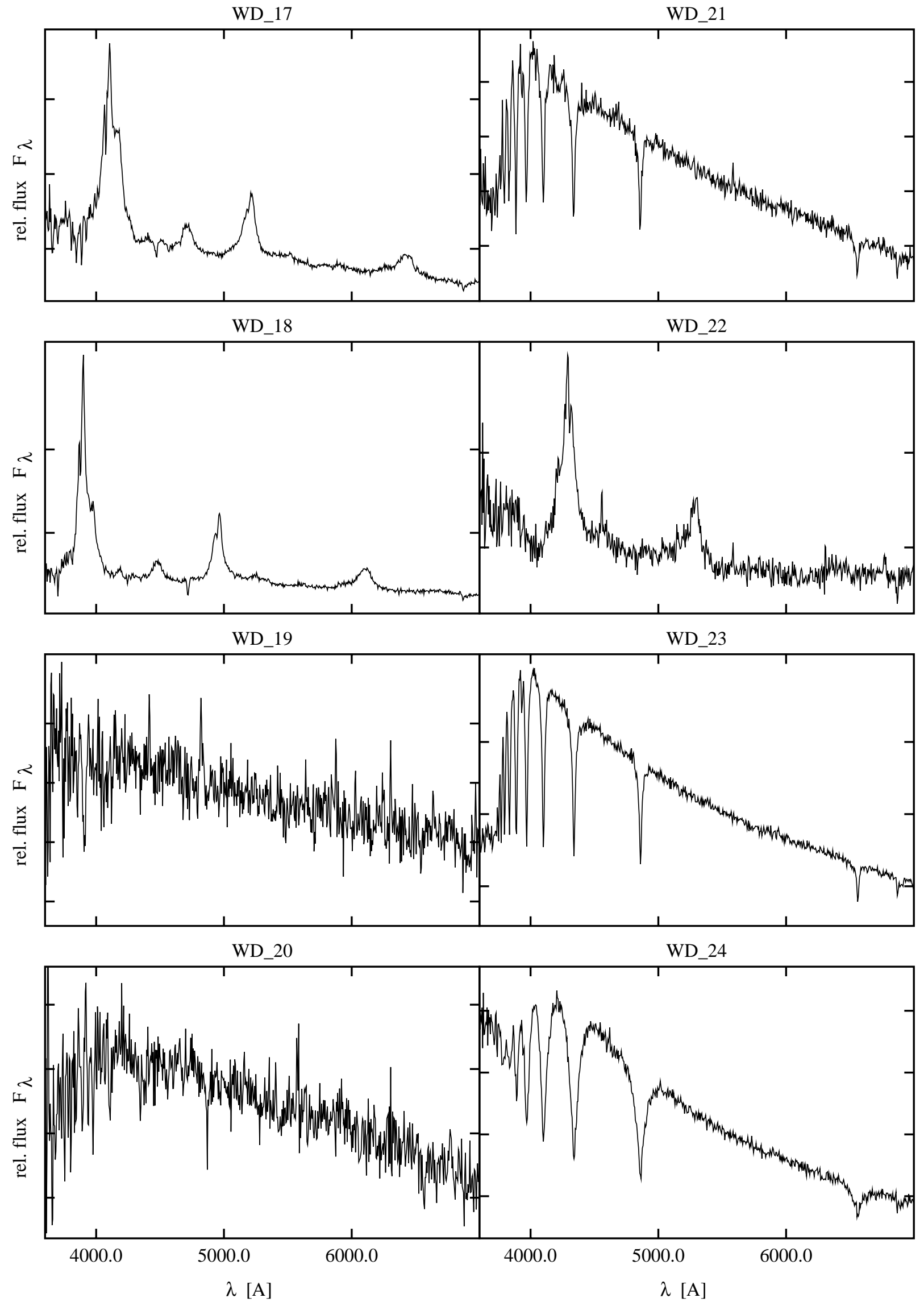

Fig. 4. VLT/FORS1 spectra of white dwarf candidates. 

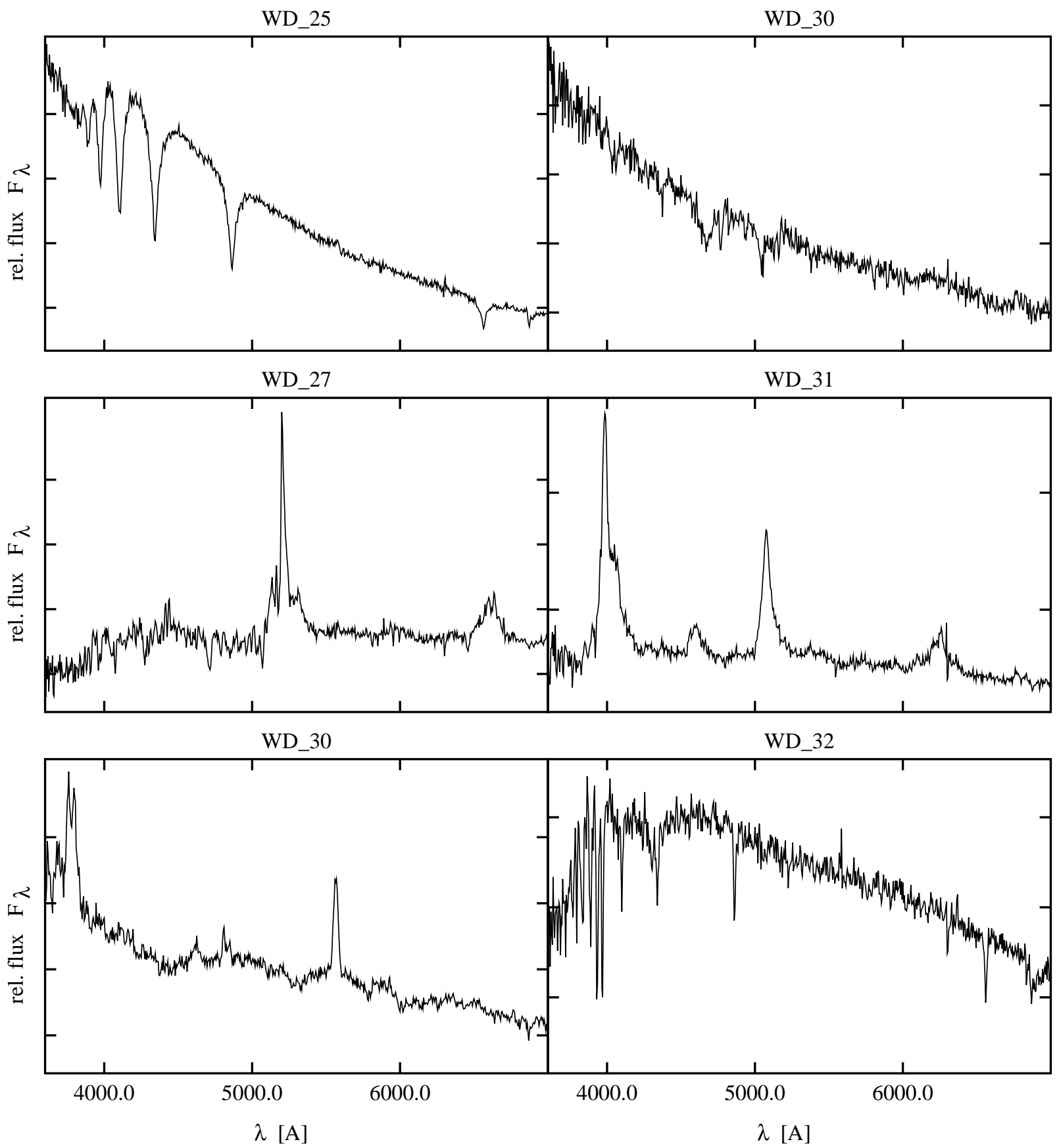

Fig. 5. VLT/FORS1 spectra of white dwarf candidates.

more recently the Hamburg Quasar Survey (Hagen et al. 1995; Homeier et al. 1998), Hamburg-ESO Quasar Survey (Wisotzki et al. 1996; Wisotzki et al. 2000; Christlieb et al. 2001), Montreal-Cambridge-Tololo Survey (Lamontagne et al. 2000), and Edinburgh-Cape Survey (Kilkenny et al. 1997). As has been pointed out by Boyle (1989), these surveys are confined to white dwarfs within $100-200$ pc of the sun and do not yield information on the scale height of the distribution. His study of $41 \mathrm{DA}$ white dwarfs with estimated $>80 \%$ completeness down to $B=20.4$ provided the first reliable estimate of the scale height from a spectroscopically confirmed sample, resulting in $275 \pm 50 \mathrm{pc}$. The area of the EIS-WIDE survey is smaller by about a factor of 10 compared to Boyle, but the limiting magnitude ( $\sim B=21.8$, see below) is fainter, thus reaching more distant white dwarfs. This provided our motivation to explore the possible use of the EIS catalog for constraints on the local space density and scale height of the white dwarf population. In this paper we report the results of our spectroscopic identification for the 32 objects of the EIS catalog with the ESO Very Large Telescope.

\section{The EIS sample of white dwarf candidates}

The four patches of the EIS-wide survey (Nonino et al. 1999) were selected because of low optical extinction, low 
FIR emission, and low H I column density. They cover a range of galactic latitudes of possible interest for galactic studies. Patch B is a 1.7 square degree region near the South Galactic Pole. Due to the bad weather conditions caused by El Niño during the survey only patch B could be observed in all three $B V I$ filter bands.

The observations of patch B were carried out over several months in the period July 1997 to December 1997 with the EMMI camera of the $3.5 \mathrm{~m}$ New Technology Telescope at La Silla (Prandoni et al. 1999). The effective field-of-view is about $9^{\prime} \times 8.5^{\prime}$. The observations were conducted using a sequence of $150 \mathrm{~s}$ exposures shifted by half of the size of an EMMI field both in right ascension and declination. Each position of the sky has, therefore, been observed with a total integration time of $300 \mathrm{~s}$. In addition, photometric and spectrophotometric standards have been regularly measured during EIS nights.

The survey uses a special set of $B V I_{\mathrm{c}}$ filters with higher transmission than the Johnson-Cousins filters. The effective wavelengths are close to the Johnson-Cousins values but the EIS passbands are broader and have sharper cutoffs. A color transformation between the filter sets has been determined from standard star observations during the survey (Prandoni et al. 1999).

Source detection and extraction has been performed with routines of the SExtractor software package of Bertin \& Arnouts (1996). The completeness of the survey could be determined by using a single field where several exposures have been made over the period of observations of patch B. The co-added exposures reach at least one magnitude deeper than the survey observations. A comparison shows that the survey is $80 \%$ complete at $B \sim 24$, $V \sim 23.5$, and $I \sim 22.5$ (Prandoni et al. 1999).

Zaggia et al. (1999) have defined a sample of pointlike sources from patch B. The target selection was based on reliable $I$-band detections $\left((S / N)_{I} \gtrsim 5\right)$ and avoided regions observed during periods with very poor seeing and low transparency. This reduced the used area to 1.27 sq degrees. The sample includes only objects detected in the $I$-band with $I<23$ and with the SExtractor stellarity index $\geq 0.75$. This star/galaxy separation is reliable for objects brighter than $I \sim 21.5$. The errors in magnitude for this sample are less than 0.15 mag for sources brighter than the separation limit and are about 0.4 mag near the limiting magnitude.

Figure 1 shows the "upper left" corner of the two-color diagram of the 3233 point-like sources which were simultaneously detected in $B, V$, and $I$. Zaggia et al. (1999) have compared the observed distribution with the locations of main sequence, red and sub giant stars from the stellar population models for an old halo and for a young disk by Bertelli et al. (1994), the location of the cooling sequence of white dwarfs with pure hydrogen atmospheres from Bergeron et al. (1995), and the color track for QSOs at different redshifts. This comparison led to the definition of a region with $V-I \leq 0.5$ and $B-V \leq 0.5$ for white dwarf candidates. Zaggia et al. pointed out that this region may still be contaminated by QSOs and blue horizontal
Table 1. Test of the limiting $I$ magnitude using the average $\overline{V / V_{\max }}$ for all objects and for the subgroup of the 11 quasars.

\begin{tabular}{llllll}
\hline & \multicolumn{5}{c}{$\overline{V / V_{\max }}$} \\
$I_{\text {lim }}$ & 21.1 & 21.2 & 21.3 & 21.4 & 21.5 \\
\hline 32 objects & 0.60 & 0.52 & 0.46 & 0.39 & 0.35 \\
11 quasars & 0.61 & 0.53 & 0.46 & 0.40 & 0.35 \\
\hline
\end{tabular}

branch stars, although the latter would be at distances of about $100 \mathrm{kpc}$ for $V>20$.

The list of white dwarf candidates comprises 32 objects. Figure 1 shows these candidates together with the selection criteria (dotted lines) and our own theoretical colors for DA white dwarfs with surface gravities $\log g$ of 7.0, 8.0, 9.0 and temperatures $T_{\text {eff from }} 6000$ to $80000 \mathrm{~K}$ (continuous lines). The short dotted lines are lines of constant $T_{\text {eff }}$. These colors have been calculated using our grid of theoretical model atmospheres, which have been described in Finley et al. (1997) and Homeier et al. (1998).

The location of the theoretical colors confirms that the selection criteria are well chosen to separate the blue white dwarfs from the large number of normal red main sequence stars. However, the theoretical lines also demonstrate that only fairly hot white dwarfs with effective temperatures above $8000 \mathrm{~K}$ can be identified in this diagram; there is no chance to find any very old and therefore cool halo white dwarfs in this sample.

According to the study of Zaggia et al. (1999) the star/galaxy separation is reliable for objects brighter than $I=21.5$. Only 4 of the $32 \mathrm{WD}$ candidates are slightly fainter than this value; we therefore expect that the limiting magnitude for completeness is slightly brighter than $I=21.5$. We have tested this independently by estimating the average value of $V / V_{\max }$, the volume to the distance of the individual objects divided by the maximum volume in which the object could be detected with a given limiting magnitude (Schmidt 1968; Wood \& Oswalt 1998). For a homogenous distribution of sources the average should be 0.5 . We have calculated this test assuming different values for the limiting $I$ magnitude $I_{\lim }$ around the expected value; Table 1 gives the results. Using all 32 candidates the average is about 0.5 for a limiting magnitude of $I=21.2$, close to the expected value. Since the white dwarfs in this catalog could possibly show the effects of a finite scale height - estimated at approximately 275 pc (Boyle 1989; Downes 1986) - with decreasing space density above the galactic plane, we have repeated the test using only the 11 quasars identified in this project, which gives the same result. Repeating a similar test for $B$ and $V$ magnitudes, we conclude that the candidate list with the selection boundaries as chosen by the EIS team is complete to the approximate limits of $I=21.2, V=21.6, B=21.8$.

\section{Spectroscopic observations}

The white dwarf candidates have been observed in service mode with the Antu telescope (UT1) of the 
Table 2. EIS white dwarf candidates. The first column gives the number as given in the EIS catalog (WD_1 - WD_32). Coordinates and magnitudes were obtained from the EIS webpage, the types of the objects have been determined from our spectroscopic observations: DA, DQ, DC = white dwarfs; QSO = quasars (with redshifts given); FHB = field horizontal branch stars; em ? = peculiar object, see text.

\begin{tabular}{|c|c|c|c|c|c|c|c|c|}
\hline WD & Name & $\alpha(\mathrm{J} 2000)$ & $\delta(\mathrm{J} 2000)$ & $I$ & $B-V$ & $V-I$ & Type & $z$ \\
\hline 1 & EIS 0044-2914 & 004503.90 & -291416.9 & 18.390 & 0.316 & 0.341 & FHB & \\
\hline 2 & EIS $0045-2947$ & 004545.94 & -294736.3 & 18.927 & 0.316 & 0.402 & QSO & 1.39 \\
\hline 3 & EIS $0045-2917$ & 004546.93 & $-29 \quad 1730.2$ & 20.554 & 0.475 & 0.371 & FHB? & \\
\hline 4 & EIS $0047-2922$ & 004713.29 & -292252.4 & 21.043 & 0.199 & 0.301 & $\mathrm{DA}$ & \\
\hline 5 & EIS 0047-2925 & 004741.21 & -292536.8 & 21.548 & 0.167 & 0.431 & QSO & 2.13 \\
\hline 6 & EIS $0047-2926$ & 004746.22 & -292639.5 & 20.963 & 0.392 & 0.481 & FHB & \\
\hline 7 & EIS $0048-2942$ & $0048 \quad 01.88$ & -294202.3 & 19.471 & 0.159 & 0.290 & FHB & \\
\hline 8 & EIS 0048-2939 & 004806.62 & -293918.0 & 19.840 & -0.042 & -0.067 & DA & \\
\hline 9 & EIS 0048-2924 & $0048 \quad 15.99$ & -292416.8 & 19.460 & 0.381 & 0.404 & QSO & 1.54 \\
\hline 10 & EIS 0048-2939 & 004820.09 & -293936.7 & 20.834 & 0.081 & 0.268 & FHB & \\
\hline 11 & EIS $0048-2929$ & 004842.47 & -292934.6 & 18.340 & 0.254 & 0.404 & $\mathrm{DA}$ & \\
\hline 12 & EIS $0048-2918$ & 004844.90 & -291824.0 & 19.963 & 0.470 & 0.469 & FHB & \\
\hline 13 & EIS 0048-2940 & 004851.47 & -294038.6 & 21.619 & 0.079 & 0.218 & $\mathrm{DC}$ & \\
\hline 14 & EIS 0048-2924 & 004853.04 & -292437.5 & 20.609 & 0.397 & 0.479 & FHB & \\
\hline 15 & EIS $0048-2943$ & 004859.20 & -294302.3 & 17.895 & 0.178 & 0.302 & $\mathrm{DA}$ & \\
\hline 16 & EIS 0049-2936 & 004908.37 & -293618.4 & 19.751 & 0.448 & 0.368 & QSO & 1.48 \\
\hline 17 & EIS 0049-2939 & 004911.34 & -293933.1 & 19.168 & 0.124 & 0.452 & QSO & 2.37 \\
\hline 18 & EIS 0049-2926 & 004924.64 & -292659.4 & 19.942 & 0.275 & 0.341 & QSO & 2.20 \\
\hline 19 & EIS 0049-2938 & 004927.36 & -293856.4 & 21.879 & 0.259 & 0.237 & em? & \\
\hline 20 & EIS 0049-2914 & 004930.88 & -291433.6 & 20.991 & 0.326 & 0.444 & FHB & \\
\hline 21 & EIS 0049-2941 & 004943.17 & -294105.3 & 20.490 & 0.190 & 0.286 & $\mathrm{FHB}$ & \\
\hline 22 & EIS 0049-2932 & 004954.72 & -293210.0 & 20.869 & 0.073 & 0.458 & QSO & 1.77 \\
\hline 23 & EIS 0049-2916 & 004958.70 & $-2916 \quad 15.4$ & 19.528 & 0.191 & 0.220 & FHB & \\
\hline 24 & EIS 0050-2924 & 005030.48 & -292424.8 & 20.413 & 0.101 & -0.106 & $\mathrm{DA}$ & \\
\hline 25 & EIS 0050-2922 & 005037.90 & -292245.6 & 18.957 & -0.007 & -0.258 & DA & \\
\hline 26 & EIS 0050-2920 & 005049.29 & -292047.1 & 20.655 & 0.292 & 0.496 & & \\
\hline 27 & EIS 0050-2914 & 005055.45 & -291440.6 & 21.618 & 0.023 & 0.266 & QSO & 3.29 \\
\hline 28 & EIS 0051-2933 & 005100.78 & -293326.1 & 18.749 & 0.298 & 0.385 & QSO & \\
\hline 29 & EIS 0051-2936 & 005100.94 & -293615.6 & 19.997 & 0.376 & 0.462 & QSO & 1.42 \\
\hline 30 & EIS 0051-2932 & 005156.64 & -293223.5 & 20.972 & 0.073 & 0.076 & DQ & \\
\hline 31 & EIS 0052-2938 & 005236.64 & -293840.6 & 21.236 & 0.176 & 0.438 & QSO & 2.28 \\
\hline 32 & EIS $0053-2932$ & $0053 \quad 42.87$ & -293227.0 & 20.418 & 0.409 & 0.412 & FHB & \\
\hline
\end{tabular}

ESO Very Large Telescope between June 24 and August 28, 2000. The FORS1 (Focal Reducer/Low Dispersion Spectrograph) instrument was used with the $300 \mathrm{~V}$ grism, the order separation filter GG375+30, and a $1^{\prime \prime}$ slit. The resulting resolution is about $12 \AA$ in the range from $3600 \AA$ to $7300 \AA$. The exposure times were chosen to obtain a signal-to-noise ratio of 25 per pixel and ranged from 2 to $112 \mathrm{~min}$.

The observations were reduced with standard procedures of the IRAF package. We have used the master bias and flat field images as provided routinely by the FORS calibration plan. The wavelength scale was obtained with exposures of $\mathrm{He}, \mathrm{Ar}$, and $\mathrm{HgCd}$ arc lamps. An observation of the standard star GD 50 has been used for the flux calibration.

The complete list of white dwarf candidates is given in Table 2. The resulting spectra are plotted in Figs. 2 to 5. The known quasar WD_28 was not included in our campaign; WD_26 could not be scheduled during the service mode observations.
Table 3. Atmospheric parameters, absolute visual magnitude and distance for the identified white dwarfs. WD_30 is of spectral type DQ, WD_13 is a DC - their atmospheric parameters are estimates. All others are DA and have been fitted with a DA model grid.

\begin{tabular}{rrrrrrr}
\hline WD & $T_{\text {eff }}$ & $\log g$ & $M_{V}$ & $V$ & $d[\mathrm{pc}]$ & age [Gyr] \\
\hline 4 & 8890 & 8.29 & 13.1 & 21.3 & 437 & 1.326 \\
8 & 17210 & 7.75 & 10.7 & 19.8 & 661 & 0.095 \\
11 & 7960 & 7.99 & 13.1 & 18.7 & 132 & 1.138 \\
13 & 10000 & 8.00 & 12.3 & 21.8 & 794 & 0.880 \\
15 & 8980 & 8.17 & 12.9 & 18.2 & 115 & 1.106 \\
24 & 13300 & 7.67 & 11.1 & 20.3 & 692 & 0.202 \\
25 & 20100 & 7.84 & 10.6 & 18.7 & 417 & 0.060 \\
30 & 10500 & 8.00 & 12.1 & 21.0 & 575 & 0.750 \\
\hline
\end{tabular}

\section{Results and discussion}

As expected, the white dwarf candidate sample is contaminated by a number of quasars in the same area of the $B V I$ two-color diagram, which are easily recognized from 


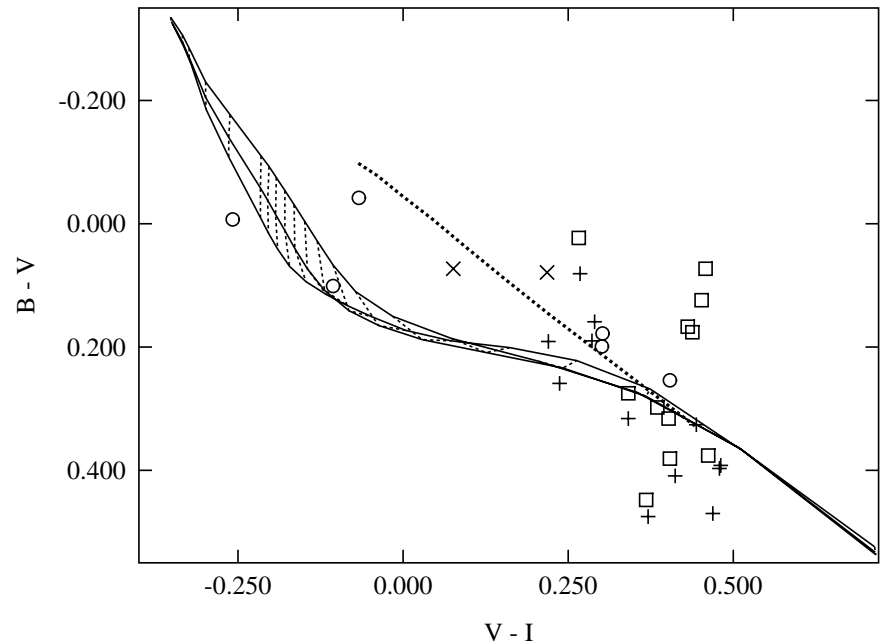

Fig. 6. Two-color diagram of EIS WD candidates. Circles: confirmed DA white dwarfs; $\times$ : DQ/DC white dwarfs; squares: quasars; crosses: other field stars.

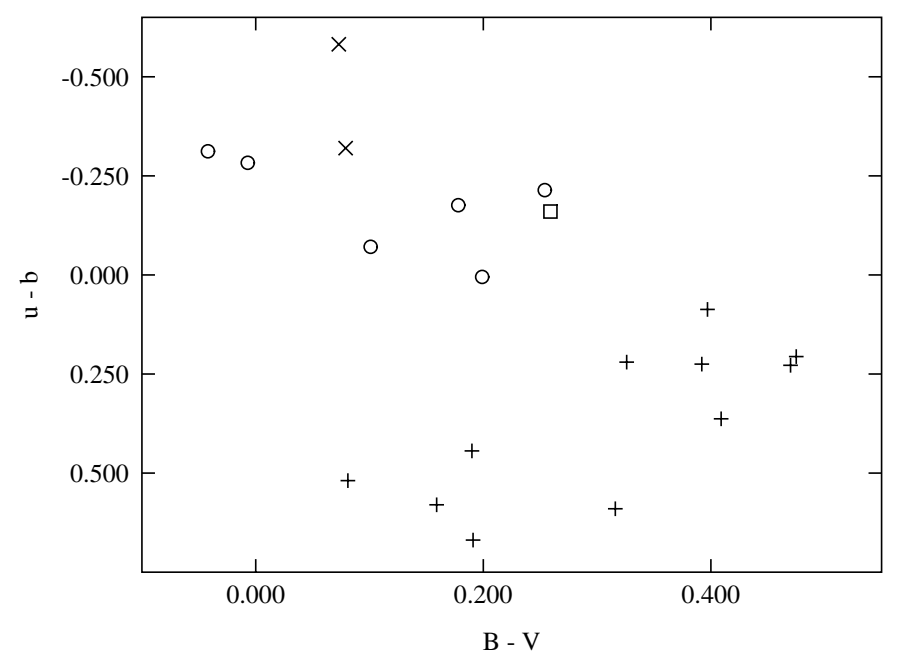

Fig. 7. Two-color diagram of EIS WD candidates. Circles: confirmed DA white dwarfs; $\times$ : DQ/DC white dwarfs; square: stellar object with blue continuum and emission lines; crosses: field horizontal branch stars.

the broad emission lines. Somewhat surprising is the presence of a quasar with $z=3.29$ (WD_27) in the sample. With this redshift and also according to the spectral energy distribution (we have two independent, but very similar spectra) it should be redder than the sample limits, whereas according to the observed colors it is the bluest quasar of the whole sample. However, WD_27 is one of the faintest candidates and the one with the largest photometric errors $(0.220$ in $B-V$ and 0.140 in $V-I)$. In addition - although we have not been able to verify that conclusively from the EIS papers - it is very likely that the passbands have been observed in different nights, since the given distribution of seeing conditions differs quite significantly between filters (Prandoni et al. 1999). Luminosity variations of the quasar could thus influence the measured colors.
WD_8, WD_24, and WD_25, are clearly normal DA white dwarfs. The spectrum of WD_30 is very similar to the known DQ white dwarf G 268-40. Koester et al. (1982) have determined $T_{\text {eff }}=10500 \mathrm{~K}$ and $\mathrm{C} / \mathrm{He}=2 \times 10^{-3}$ for this object. We assume in the following their result also for WD_30.

WD_13 shows no obvious lines, but a blue continuum up to the Balmer jump near the short wavelength end of our spectra. We tentatively identify this object as a DC white dwarf and estimate the effective temperature from the colors and the non-visibility of He lines at about $10000 \mathrm{~K}$. Most of the remaining objects are obviously stellar, with fairly narrow Balmer lines, and often strong CaII resonance lines.

All stellar objects with Balmer lines have been analyzed with our $\chi^{2}$ fitting techniques as described in (Homeier et al. 1998). The theoretical model grid for DA white dwarfs included models with surface gravities between $\log g=6.0-9.0$ and effective temperatures from $6000 \mathrm{~K}$ to $100000 \mathrm{~K}$. In addition to the clear DA white dwarfs identified immediately (WD_8, WD_24, and WD_25) the fitting routine determines solutions in the white dwarf regime of parameters for WD_4, WD_11, and WD_15. The parameters of these objects are given in Table 3, where the cooling ages have been determined from the tracks of Wood (1995) for "thick" hydrogen envelopes for the DA and "thin" envelopes for the DQ and DC. The formal errors of the $\chi^{2}$ fitting technique range from $40 \mathrm{~K} / 0.06$ for $T_{\text {eff }}$ and $\log g$ for the brighter objects to typically $330 \mathrm{~K} / 0.25$ for the fainter ones.

No solutions for spectral fits are found - within our model grid - for the remaining stellar objects. We identify these other objects (with the exception of WD_19, see below) with lower gravity field horizontal branch stars and possibly other A-type stars, which cannot be distinguished at this resolution as described e.g. in Wilhelm et al. (1999). This classification is uncertain for WD_3, which has a very noisy spectrum. We cannot exclude that the object might be a very cool DA, although in the twocolor-diagram (Fig. 7, see below) it is the reddest object in $B-V$ and in a similar position as other FHB stars.

Figure 6 shows that the 3 hot DA fall indeed in the expected region of the $(B-V)-(V-I)$ two-color diagram, and the two helium-rich white dwarfs into the vicinity of the theoretical DB/DC colors (thick dotted line). The cooler DA candidates, however, are indistinguishable in this diagram from the other halo stars.

In order to gain some insight into the results of the spectral fitting routine - to identify the spectral features, which distinguish the high-gravity objects - we have tried to separate cool white dwarfs and lower gravity objects on the basis of equivalent widths of Balmer lines, with no convincing success. The one feature, however, which clearly separates the two groups, is the Balmer jump and the overlapping of the higher Balmer lines near the series limit. While the objects identified by us as cool DA show Balmer lines up to $\mathrm{H} 8$ or $\mathrm{H} 9$ only, in all other objects the lines are visible to at least H13. 


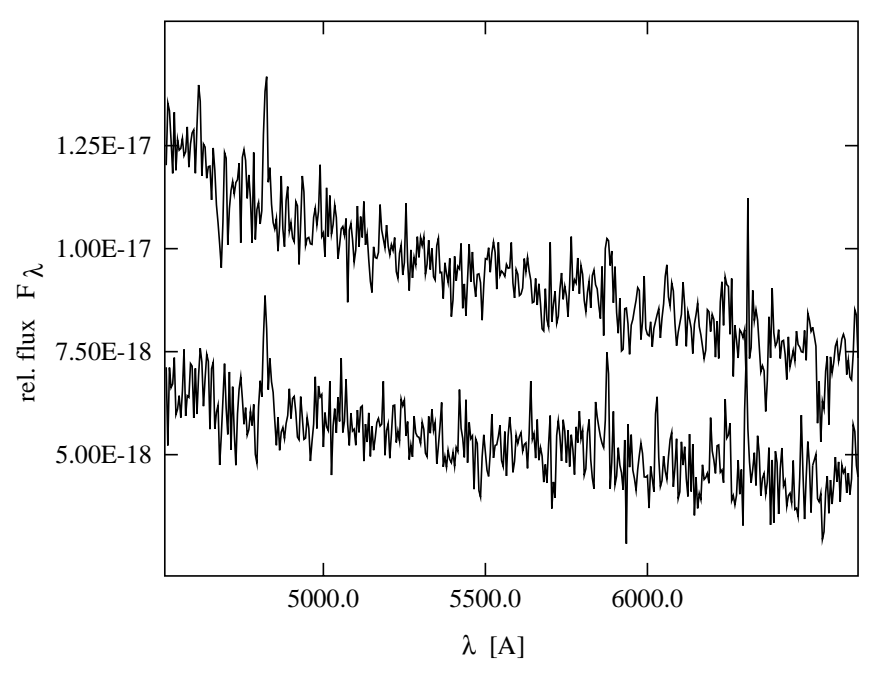

Fig. 8. Part of two independent spectra for WD_19, showing emission features. The upper spectrum has been shifted by $2 \times 10^{-18}$ for clarity.

In order to find a quantitative measurement for the blue continuum near the short wavelength end of the spectra, we have defined artificial $u$ and $b$ magnitudes and a color $u-b$ by integrating the spectral flux in the wavelength regions from $3600-3720$ and $4500-4650 \AA$. The twocolor diagram using this color and $B-V$ is shown in Fig. 7; it clearly shows the separation of white dwarfs and lower gravity objects. We emphasize that this exercise with equivalent widths and artificial colors is not used for the parameter determination of the white dwarfs, which is based on detailed spectral fitting of atmospheric models. Figure 7 is only meant to demonstrate that even without this fitting procedure different stellar groups can be distinguished directly based on a simple "classification" of the spectra.

The one remaining peculiar object is WD_19. This object shows a very strange spectrum with an absorption line at $6540 \AA$, possibly $\mathrm{H} \alpha$ shifted and split in several components, a weak emission feature at $6641 \AA$, an emission line at $4823 \AA$, and possibly some other absorption lines below $4000 \AA$. We have two independent spectra of this object (Fig. 8) and the features are present in both. Because the continuum is also very blue with no decline towards the Balmer jump, this may be a degenerate object, possibly with a companion or accretion disk. With the present observations we are unable to find a clear identification.

Our final conclusion is that 8 of the 32 original candidates are spectroscopically confirmed white dwarfs. Since the statistical population properties demand as a minimum the determination of a local number density and a scale height, even if we neglect complications such as possible contributions from a thick disk or halo, this number is clearly too small to draw any firm conclusions from this sample.

Using the $1 / V_{\max }$ method in an extremely simplified version - neglecting the density variation with distance from the galactic plane - to estimate a space density from the individual contributions of the 8 white dwarfs of Table 3, we obtain $2.1 \times 10^{-5} \mathrm{pc}^{-3}$. Since our sample is limited to effective temperatures hotter than about $8000 \mathrm{~K}$ and correspondingly younger than about $1.3 \times 10^{9}$ years, which excludes the majority of white dwarfs according to the standard luminosity function, this number is not inconsistent with the results $0.003 \mathrm{pc}^{-3}$ of Liebert et al. (1989), but does not add significant new information about the statistical properties of white dwarfs.

The group of metal poor halo A stars found in this study is potentially of more interest than the white dwarfs. Their distances above the galactic plane must be of the order of $50 \mathrm{kpc}$ and a study of their kinematic properties could probe the mass distribution of the outer halo. Unfortunately the determination of radial velocities with the required accuracy will require a very large telescope.

Acknowledgements. This work has been supported by the Deutsches Zentrum für Luft- und Raumfahrt (DLR) under grant 50 OR 96173.

\section{References}

Bergeron, P., Wesemael, F., Lamontagne, R., et al. 1995, ApJ, 449, 258

Bertelli, G., Bressan, A., Chiosi, C., Fagotto, F., \& Nasi, E. 1994, A\&AS, 106, 275

Bertin, E., \& Arnouts, S. 1996, A\&AS, 117, 393

Boyle, B. J. 1989, MNRAS, 240, 533

Christlieb, N., Wisotzki, L., Reimers, D., et al. 2001, A\&A, 366,898

Downes, R. A. 1986, ApJS, 61, 569

Finley, D. S., Koester, D., \& Basri, G. 1997, ApJ, 488, 375

Green, R. F., Schmidt, M., \& Liebert, J. 1986, ApJS, 61, 305

Hagen, H.-J., Groote, D., Engels, D., \& Reimers, D. 1995, A\&AS, 111, 195

Homeier, D., Koester, D., Hagen, H. J., et al. 1998, A\&A, 338, 563

Kilkenny, D., O’Donoghue, D., Koen, C., Stobie, R. S., \& Chen, A. 1997, MNRAS, 287, 867

Koester, D., Weidemann, V., \& Zeidler, E. 1982, A\&A, 116, 147

Lamontagne, R., Demers, S., Wesemael, F., Fontaine, G., \& Irwin, M. J. 2000, AJ, 119, 241

Liebert, J., Dahn, C. C., \& Monet, D. G. 1989, in White Dwarfs, IAU Colloq., 114, 15

Nonino, M., Bertin, E., da Costa, L., et al. 1999, A\&AS, 137, 51

Prandoni, I., Wichmann, R., da Costa, L., et al. 1999, A\&A, 345,448

Renzini, A., \& Da Costa, L. N. 1997, The Messenger, 87, 23

Schmidt, M. 1968, ApJ, 151, 393

Wilhelm, R., Beers, T. C., Sommer-Larsen, J., et al. 1999, AJ, 117, 2329

Wisotzki, L., Christlieb, N., Bade, N., et al. 2000, A\&A, 358, 77

Wisotzki, L., Koehler, T., Groote, D., \& Reimers, D. 1996, A\&AS, 115, 227

Wood, M. A. 1995, in White Dwarfs, Proc. of the 9th European Workshop on White Dwarfs Held at Kiel, Germany, 29 August-1 September 1994, XII (Springer-Verlag, Berlin Heidelberg, New York), also Lect. Notes Phys. 443, ed. D. Koester, \& K. Werner, 51

Wood, M. A., \& Oswalt, T. D. 1998, ApJ, 497, 870

Zaggia, S., Hook, I., Mendez, R., et al. 1999, A\&AS, 137, 75 